\title{
Nascent ribosomal RNA acts as surfactant that suppresses growth of fibrillar centers in nucleolus
}

\author{
Tetsuya Yamamoto ${ }^{1,2 *}$, Tomohiro Yamazaki ${ }^{*}{ }^{*}$, Kensuke Ninomiya $^{3}$, and Tetsuro Hirose ${ }^{3}$ \\ ${ }^{1}$ Institute for Chemical Reaction Design and Discovery, Hokkaido University, Kita 21, Nishi 10, Kita-ku, Sapporo, \\ 001-0021, Japan. ${ }^{2}$ PRESTO, Japan Science and Technology Agency (JST), 4-1-8, Honcho, Kawaguchi, Saitama \\ 332-0012, Japan. and ${ }^{3}$ Graduate School of Frontier Biosciences, Osaka University, Suita 565-0871, Japan.
}

Received YYYY-MM-DD; Revised YYYY-MM-DD; Accepted YYYY-MM-DD

\begin{abstract}
Liquid-liquid phase separation (LLPS) has been thought to be the assembly mechanism of the multiphase structure of nucleolus, the site of ribosomal biogenesis. Condensates assembled by LLPS increase their size to minimize the surface energy as far as their components are available. However, multiple microphases, fibrillar centers (FCs), dispersed in a nucleolus are stable and their size does not grow unless the transcription of pre-ribosomal RNA (prerRNA) is inhibited. To understand the mechanism of the suppression of the growth of FCs, we here construct a minimal theoretical model by taking into account the nascent pre-rRNA transcripts tethered to the surfaces of FCs by RNA polymerase I. Our theory predicts that nascent pre-rRNA transcripts generate the lateral osmotic pressure that counteracts the surface tension of the microphases and this suppresses the growth of the microphases over the optimal size. The optimal size of the microphases decreases with increasing the transcription rate and decreasing the rate of RNA processing. This prediction is supported by our experiments showing that the size of FCs increased with increasing the dose of transcription inhibitor. This theory may provide insight in the general mechanism of the size control of nuclear bodies.
\end{abstract}

\section{INTRODUCTION}

Nucleus is not a uniform solution of chromatin, but there are a variety of nuclear bodies, such as nucleoli $(1,2,3,4)$, transcriptional condensates $(5,6,7,8)$, and paraspeckles (9), in the inter-chromatin spaces. Many of the nuclear bodies are scaffolded by complexes between RNA and RNA-binding proteins (RBPs). A class of RNA transcripts, which are essential to form nuclear bodies, are called architectural RNA (arcRNA) $(9,10,11)$. Growing number of evidences suggest that some nuclear bodies are assembled via liquid-liquid phase separation (LLPS) due to the multi-valent interaction between RBPs bound to arcRNAs $(12,13)$. Condensates produced by LLPS are spherical and increase their size by coarsening and/or coalescence to minimize the total surface area due to the surface tension (macroscopic phase separation) (14).

Nucleolus is a nuclear body, in which pre-ribosomal RNAs (pre-rRNAs) are transcribed by RNA polymerase I (Pol I) and ribosomes are assembled $(1,2)$. A nucleolus is not a uniform disordered liquid, but multiple microphases, called fibrillar centers (FCs), are dispersed in the sea of a granular component (GC) (3), see Figure 1a. The multiphase structure of nucleolus has been thought to be assembled via simple LLPS (15). However, this picture may not be complete because the microphases do not show coalescence or coarsening to increase their sizes. Indeed, when the Pol I transcription of pre-rRNA is inhibited, FCs show coalescence, as in the case of LLPS, and are excluded to the surface of the nucleolus (16). This implies that transcription somehow suppresses the growth of the microphases. Ribosomal DNA (rDNA), from which pre-rRNAs are transcribed, is a repeat sequence of coding units $(\sim 10 \mathrm{kbps})$ intervened by the intergenic regions ( $\sim 30 \mathrm{kbps})(2)$. The active rDNA units and Pol I are localized at the surfaces of FCs; the transcription of pre-rRNAs happens at the surfaces of FCs $(3,16,17$, 18). Nascent pre-rRNA transcripts are therefore localized at the surface of each FC and forms a layer, called dense fibrillar component (DFC), in which fibrillarin (FBL) proteins and other RNA processing factors are condensed (18). FBL proteins show phase separation with the physiological concentration and bind to nascent pre-rRNA transcripts (15, 18). These experimental results imply that nascent pre-rRNA transcripts may act as 'surfactants' that suppress the growth of FCs.

We here construct a simple theoretical model that predicts the contributions of nascent pre-rRNA transcripts to the stability of FCs in a nucleolus. Our theory predicts that the nascent pre-rRNA transcripts generate the lateral pressure that counteracts with the surface tension of FCs. The size of FCs is determined by the balance of the surface tension and the lateral pressure. The lateral pressure depends on the transcription and processing of nascent pre-rRNA transcripts as well as the binding of RBPs to the nascent pre-rRNA transcripts. Our

\footnotetext{
*To whom correspondence should be addressed. (Theory: T. Yamamoto) Tel: +81-11-706-9665; Email: tyamamoto@icredd.hokudai.ac.jp, (Experiment: T. Yamazaki) Tel: +81-6-6879-4675; Email: tyamazaki@fbs.osaka-u.ac.jp

This is an Open Access article distributed under the terms of the Creative Commons Attribution Non-Commercial License (http://creativecommons.org/licenses/ by-nc/2.0/uk/) which permits unrestricted non-commercial use, distribution, and reproduction in any medium, provided the original work is properly cited.
} 
a.

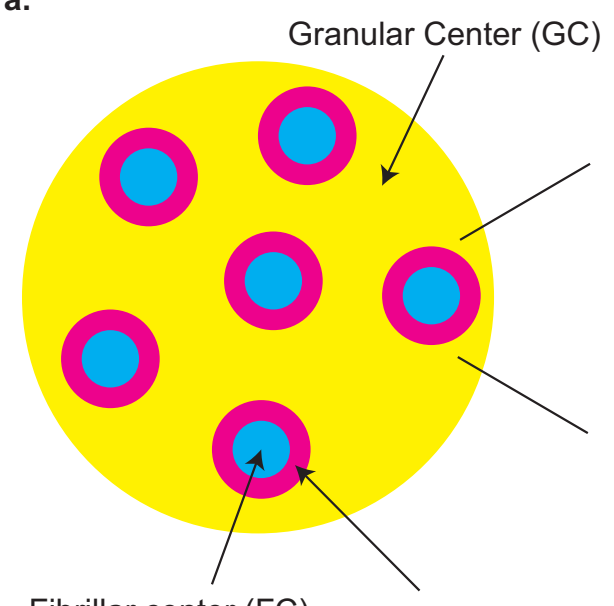

Fibrillar center (FC)

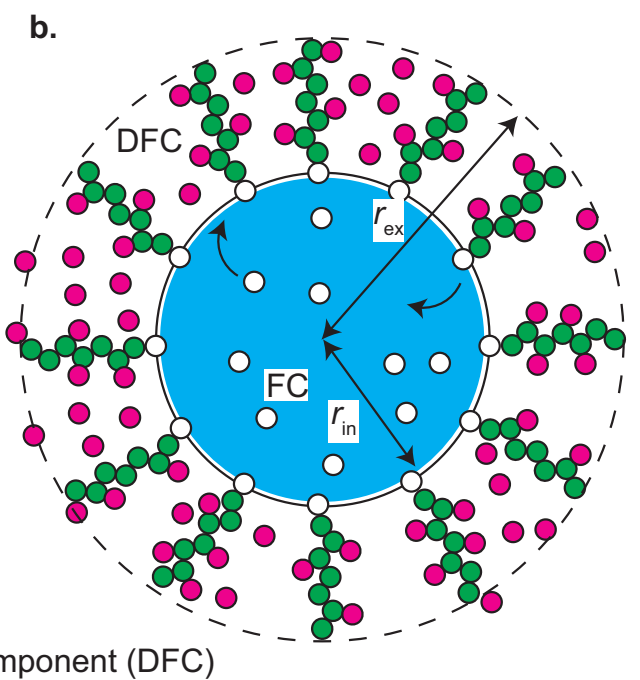

Figure 1. Model of multi-phase structure of a nucleolus. a. A nucleolus is composed of multiple fibrillar center (FC) microphases in the sea of the granular component (GC). There is a layer of dense fibrillar center (DFC) between each FC and GC. b. RNA polymerase I (Pol I) molecules (white particles) are entrapped in FC microphases (light blue) and the active rDNA units (black line) are localized at the surfaces of microphases. Nascent pre-rRNA transcripts (green particles) are thus at the surfaces of the microphase and form a DFC layer with RNA-binding proteins (magenta particles). The interface between FC and DFC is located at a distance $r_{\text {in }}$ from the center and the interface between DFC and GC is located at a distance $r_{\text {ex }}$.

theory predicts that the size of FCs decreases with increasing the transcription rate and the average time of RNA processing. The suppression of the growth of FCs by the complexes of nascent pre-rRNA and RBPs results from the fact that these complexes are end-grafted to the surfaces of FCs via Pol I. It is in contrast to many other biological condensates, where RNA transcripts diffuse freely. To test our prediction, we experimentally measured the size of FCs by changing the dose of BMH-21, which specifically inhibits the transcription of Pol I. The size of FCs increased continuously and monotonically with increasing the dose of BMH-21, in agreement with our theoretical prediction.

\section{MATERIALS AND METHODS}

\section{Nascent RNA brush model}

We here construct a minimal model of a nucleolus, in which multiple spherical FC microphases are dispersed, see Figure 1. Pol I molecules (shown by white particles in Figure 1b) are entrapped in the microphases (shown by a light blue droplet in Figure 1b). The active repeat units of rDNA (shown by the black line in Figure 1b) are localized at the surfaces of the microphases. The number of Pol I molecules $N_{\mathrm{pl}}$ and the number of active rDNA units $N_{\mathrm{a}}$ in the nucleolus are constant. Nascent RNA transcripts (shown by chains of green particles in Figure 1b) are localized at the surfaces of microphases and form a DFC layer with RBPs (shown magenta particles in Figure 1b). RBPs diffuse in the DFC layer when they are not bound to nascent RNA transcripts. The interface between FC and DFC is located at a distance $r_{\text {in }}$ from the center and the interface between DFC and GC is located at a distance $r_{\mathrm{ex}}$ from the center. We assume that all of the microphases in the nucleolus have equal volume and the sum of the volumes of microphases is fixed to $V_{\mathrm{m}}$. We analyze the volume of each microphase in the steady state.

\section{Transcription and RNA processing}

The number of nascent pre-rRNA transcripts at the surface of each microphase is determined by the transcription dynamics and the dynamics of RNA processing. We use an extension of the model used by Stasevich and coworkers (19) to treat the transcription dynamics, see Figure 2. Pol I in a microphase binds to the transcription start site (TSS) of an active rDNA unit. The Pol I bound to the TSS starts transcription (Figure 2b) or returns to the microphase without starting transcription (Figure 2a). For cases in which the rate $k_{\mathrm{e}}$ with which Pol I bound to the TSS starts transcription is smaller than the rate with which this Pol I returns to the microphase without starting transcription, the occupancy of the TSS by Pol I is represented by the form $\rho /\left(\rho+K_{\mathrm{pl}}\right)$ by using the concentration $\rho$ of Pol I in the microphase and the equilibrium constant $K_{\mathrm{pl}}$. The Pol I that has started transcription synthesizes a prerRNA transcript while it moves uni-directionally towards the transcription termination site (TTS). The nascent pre-rRNA transcripts are subject to co-transcriptional processing and are released to GC at the average processing time $\tau_{\mathrm{pr}}$, see Figure 2c. This treatment emphasizes the RNA region that is localized at the DFC layer and is released by the RNA processing. Other RNA regions are treated only implicitly. Pol I reaches TTS at the average elongation time $\tau_{\mathrm{e}}$ and is then released to the microphase $\left(\tau_{\mathrm{pr}}<\tau_{\mathrm{e}}\right)$, see Figure $2 \mathbf{d}$.

The concentration $\rho$ of Pol I in a microphase is determined by the relationship

$$
k_{\mathrm{e}} \frac{\rho}{\rho+K_{\mathrm{pl}}} \tau_{\mathrm{e}} N_{\mathrm{a}}+\rho V_{\mathrm{m}}=N_{\mathrm{pl}} .
$$

The first term in the left side of eq. (1) is the number of Pol I during transcription and the second term in the left side of eq. (1) is the number of Pol I diffusing in microphases. Eq. (1) predicts that the concentration $\rho$ of Pol I in microphases does not depend on the size of each microphase, but only on their total volume $V_{\mathrm{m}}$. 

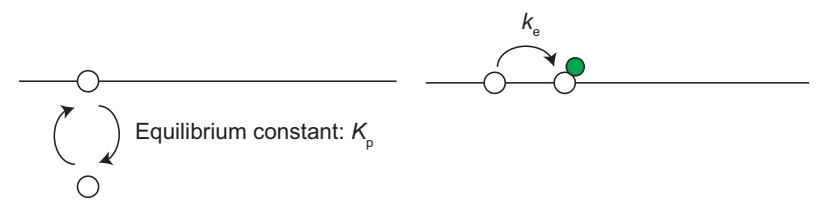

c. RNA processing $\left(t=\tau_{\mathrm{p}}\right)$

d. Termination $\left(t=\tau_{\mathrm{e}}\right)$

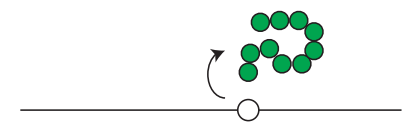

Figure 2. Transcription model. RNA polymerase I (Pol I) in a microphase binds to the transcription starting site (TSS) of an active rDNA repeat unit (a). The bound Pol I starts transcription with the rate $k_{\mathrm{e}}(\mathbf{b})$, otherwise, returns to the microphase without starting transcription (a). During the transcription, Pol I migrates uni-directionally from the TSS to the transcription terminating site (TTS) and the nascent pre-ribosomal RNA (pre-rRNA) transcripts produced in this process are subject to the co-transcriptional RNA processing (c). The nascent pre-rRNA transcripts are released to GC in the (average) processing time $\tau_{\mathrm{pr}}(\mathbf{c})$. Pol I reaches TTS in the (average) elongation time $\tau_{\mathrm{e}}$ and then is released to the microphase (d).

The surface density of nascent pre-rRNA transcripts (the number of nascent pre-rRNA transcripts per unit area) has the form

$\sigma=\frac{1}{3} k_{\mathrm{e}} \tau_{\mathrm{pr}} \frac{\rho}{\rho+K_{\mathrm{pl}}} \frac{N_{\mathrm{a}}}{V_{\mathrm{m}}} r_{\mathrm{in}}$.

Eq. (2) is derived by assuming that nascent RNA transcripts are distributed uniformly at the surfaces of microphases and by using the fact that the number of active rDNA units at the surface of each microphase is $4 \pi N_{\mathrm{a}} r_{\text {in }}^{3} /\left(3 V_{\mathrm{m}}\right)$. The surface density $\sigma$ is therefore proportional to the radius $r_{\text {in }}$ of microphases and we thus treat the surface density $\sigma$ as a rescaled radius $r_{\text {in }}$ in the Results section.

\section{Free energy of the system}

We here use the mean field theory to treat nascent RNA transcripts in a DFC layer. The advantage of using this treatment is that one can use the same free energy throughout the theory and greatly simplifies the discussion (the result by using the formal treatment in the polymer physics is discussed in the Discussion section). The free energy of a DFC layer has the form

$$
\begin{aligned}
F_{\mathrm{d}}= & \int_{r_{\mathrm{in}}}^{r_{\mathrm{ex}}} d r \frac{4 \pi r^{2}}{b^{3}}\left[f_{\text {ela }}+f_{\text {mix }}+f_{\text {int }}+f_{\text {bnd }}\right. \\
& \left.-\mu_{\mathrm{p}}\left(\phi_{\mathrm{p}}+\alpha_{\mathrm{p}} \phi_{\mathrm{r}}\right)+\Pi b^{3}\right],
\end{aligned}
$$

where $r$ is the distance from the center, see Figure $1 \mathbf{b}$. This free energy is composed of 4 contributions: $f_{\text {ela }}$ is the elastic free energy density of nascent RNA transcripts, $f_{\text {mix }}$ is the free energy density due to the mixing entropy of RBPs and solvent molecules, $f_{\text {int }}$ is the free energy density due to the interactions between RBPs, and $f_{\text {bnd }}$ is the free energy density due to the binding of RBPs to nascent RNA transcripts. $\mu_{\mathrm{p}}$ is the chemical potential of RBPs and $\Pi$ is the osmotic pressure. $\mu_{\mathrm{p}}$ and $\Pi$ play the role in the Lagrange multipliers that ensure that the number of RBPs in the system and the volume of the system are constant.

The exterior radius $r_{\text {ex }}$ (see also Figure 1b) is determined by the relationship

$\int_{r_{\mathrm{in}}}^{r_{\mathrm{ex}}} d r 4 \pi r^{2} \phi_{\mathrm{r}}=4 \pi r_{\mathrm{in}}^{2} \sigma N_{\mathrm{r}}$

where $N_{\mathrm{r}}$ is the average number of units in each nascent RNA transcripts. It is a mean field treatment that assumes that nascent RNA transcripts are composed of the same number of units and is justified within the Alexander-de Gennes approximation, with which the brush height is determined by the distance between neighboring grafting points and the average number of units per chain (if one neglects the fact that the lateral fluctuations of a chain composed of $N_{\mathrm{r}}$ units is limited to $\left.\sim N_{\mathrm{r}} b^{2}\right)(20,21)$. The free energy $F_{\mathrm{d}}$ is a functional of the volume fraction $\phi_{\mathrm{p}}(r)$ of RBPs, the volume fraction $\phi_{\mathrm{r}}(r)$ of nascent RNA units, and the occupancy $\alpha_{\mathrm{p}}(r)$ of nascent RNA units by RBPs, which are functions of the distance $r$ from the center $\left(r_{\mathrm{in}}<r<r_{\mathrm{ex}}\right)$.

The (entropic) elastic free energy density has the form

$\frac{f_{\text {ela }}}{k_{\mathrm{B}} T}=\frac{3}{2} \frac{b^{4}}{\phi_{\mathrm{r}}} \frac{\sigma^{2} r_{\text {in }}^{2}}{r^{4}}$

for nascent RNA transcripts on a spherical surface, see Figure 1b. $b$ is the length of each RNA unit. $k_{\mathrm{B}}$ is the Boltzmann constant and $T$ is the absolute temperature. Eq. (5) illustrates the fact that the volume fraction $\phi_{\mathrm{r}}$ decreases, and thus the elastic free energy $f_{\text {ela }}$ increases, as nascent RNA transcripts are stretched. Eq. (5) is derived in the spirit of the DaoudCotton theory (22), see sec. S1 in the Supplementary File for the derivation.

The free energy due to the mixing entropy of RBPs and solvent molecules has the form

$$
\begin{aligned}
\frac{f_{\text {mix }}}{k_{\mathrm{B}} T}= & \phi_{\mathrm{p}} \log \phi_{\mathrm{p}} \\
& +\left(1-\phi_{\mathrm{p}}-\left(1+\alpha_{\mathrm{p}}\right) \phi_{\mathrm{r}}\right) \log \left(1-\phi_{\mathrm{p}}-\left(1+\alpha_{\mathrm{p}}\right) \phi_{\mathrm{r}}\right) .
\end{aligned}
$$

The first term of eq. (6) is the free energy due to the mixing entropy of RBPs and the second term of eq. (6) is the free energy due to the mixing entropy of solvent molecules.

The free energy due to the interactions between RBPs has the form

$\frac{f_{\text {int }}}{k_{\mathrm{B}} T}=-\chi\left(\phi_{\mathrm{p}}+\alpha_{\mathrm{p}} \phi_{\mathrm{r}}\right)^{2}$,

where $\chi$ is the interaction parameter that accounts for the attractive interactions. For simplicity, we assumed that RBPs that are bound to nascent RNA transcripts are equivalent to RBPs that are freely diffusing in the DFC layer and that solvent molecules are equivalent to nascent RNA units in terms of the interactions.

The free energy due to the binding of RBPs to nascent RNA transcripts has the form

$\frac{f_{\mathrm{bnd}}}{k_{\mathrm{B}} T}=\phi_{\mathrm{r}}\left[\alpha_{\mathrm{p}} \log \alpha_{\mathrm{p}}+\left(1-\alpha_{\mathrm{p}}\right) \log \left(1-\alpha_{\mathrm{p}}\right)-\epsilon \alpha_{\mathrm{p}}\right]$, 
where $\epsilon k_{\mathrm{B}} T$ is the energy increase due to the binding of RBPs to nascent RNA transcripts. For simplicity, we assumed that each nascent RNA unit has one binding site of RBPs.

Eq. (3) returns to the free energy of binary mixture for $\sigma \rightarrow$ 0 and to the free energy of a polymer brush on a spherical surface for $\phi_{\mathrm{p}} \rightarrow 0$ (23).

The free energy $F$ of the system has the form

$\frac{F}{V_{\mathrm{m}}}=\frac{3}{4 \pi r_{\mathrm{in}}^{3}}\left[F_{\mathrm{d}}+4 \pi r_{\mathrm{in}}^{2} \gamma_{\mathrm{in}}+4 \pi r_{\mathrm{ex}}^{2} \gamma_{\mathrm{ex}}\right]$,

where we used the fact that the number of microphases in the system is $3 V_{\mathrm{m}} /\left(4 \pi r_{\text {in }}^{3}\right)$ to derive this form. $\gamma_{\text {in }}$ is the surface energy per unit area (surface tension) at the interface between FC and DFC and $\gamma_{\mathrm{ex}}$ is the surface energy per unit area (surface tension) at the interface betwen DFC and GC. The surface tensions, $\gamma_{\text {in }}$ and $\gamma_{\mathrm{ex}}$, depend on the local composition at the corresponding interfaces. The facts that the surface tension between GC and nucleosol is relative small (3) and that FC is excluded out from the nucleolus by the transcription inhibition $(3,16)$ imply that GC and FC are relatively hydrophilic. By neglecting the difference of the hydrophilicity between FC and GC, $\gamma_{\text {in }}$ and $\gamma_{\text {ex }}$ are represented by the forms

$\gamma_{\text {in }}=\gamma_{\mathrm{p}}\left(\phi_{\mathrm{p}}\left(r_{\mathrm{in}}\right)+\alpha_{\mathrm{p}}\left(r_{\mathrm{in}}\right) \phi_{\mathrm{r}}\left(r_{\mathrm{in}}\right)\right)$

$\gamma_{\mathrm{ex}}=\gamma_{\mathrm{p}}\left(\phi_{\mathrm{p}}\left(r_{\mathrm{ex}}\right)+\alpha_{\mathrm{p}}\left(r_{\mathrm{in}}\right) \phi_{\mathrm{r}}\left(r_{\mathrm{ex}}\right)\right)$,

where $\gamma_{\mathrm{p}}$ is the surface tension between a nucleosol and a liquid of the RBPs and is proportional to $\chi$.

\section{Steady state}

Minimizing the free energy with respect to $\phi_{\mathrm{p}}(r)$ and $\phi_{\mathrm{r}}(r)$ with the condition of eq. (4) leads to the chemical potential $\mu_{\mathrm{p}}$ of RBPs and the osmotic pressure $\Pi$ in the forms

$$
\begin{aligned}
\frac{\mu_{\mathrm{p}}}{k_{\mathrm{B}} T}= & \log \phi_{\mathrm{p}}-\log \left(1-\phi_{\mathrm{p}}-\left(1+\alpha_{\mathrm{p}}\right) \phi_{\mathrm{r}}\right) \\
& -2 \chi\left(\phi_{\mathrm{p}}+\alpha_{\mathrm{p}} \phi_{\mathrm{r}}\right) \\
\frac{\Pi b^{3}}{k_{\mathrm{B}} T}= & -\left(1+\alpha_{\mathrm{p}}\right) \phi_{\mathrm{r}}-\log \left(1-\phi_{\mathrm{p}}-\left(1+\alpha_{\mathrm{p}}\right) \phi_{\mathrm{r}}\right) \\
& -\chi\left(\phi_{\mathrm{p}}+\alpha_{\mathrm{p}} \phi_{\mathrm{r}}\right)^{2}-\frac{3\left(\sigma b^{2}\right)^{2}}{\phi_{\mathrm{r}}} \frac{r_{\mathrm{in}}^{4}}{r^{4}}
\end{aligned}
$$

see also sec. S2 in the Supplementary File. The chemical potential and the osmotic pressure is continuous across the interfaces at $r=r_{\mathrm{ex}}$ and $r=r_{\text {in }}$ due to the local equilibrium. Minimizing the free energy with respect to the occupany $\alpha_{\mathrm{p}}$ leads to the form

$\log \alpha_{\mathrm{p}}-\log \left(1-\alpha_{\mathrm{p}}\right)-\epsilon-1-\log \phi_{\mathrm{p}}=0$,

where we used eq. (12) to derive this relationship. The time evolution equations of the volume fractions of RBPs and nascent RNA units can be derived by using the Onsager theory (14).

With our model, the nucleolus is driven out from the thermodynamic equilibrium only by the transcription, which produces nascent RNA transcripts at the surfaces of microphases. For simplicity, we here treat the cases in which the chemical potential $\mu_{\mathrm{p}}$ of RBPs and the osmotic pressure $\Pi$ are constant throughout the DFC layer and the occupancy $\alpha_{\mathrm{p}}$ is derived by using eq. (14). These approximations are exact for cases in which the relaxation time of a nascent pre-rRNA transcript and the time scale of protein binding are both shorter than the time scale with which Pol I adds one RNA unit to the nascent RNA during transcription. The chemical potential $\mu_{\mathrm{p}}$ and the osmotic pressure $\Pi$ is determined by the composition in GC. The chemical potential has an approximate form $\mu_{\mathrm{p}}=$ $k_{\mathrm{B}} T \log \phi_{\mathrm{p} 0}$ by using the volume fraction $\phi_{\mathrm{p} 0}$ of RBPs in the nucleosol.

\section{Cell culture, drug treatment, immunofluorescence, and quantification of the size of FCs}

HeLa cells were maintained in DMEM containing high glucose (Nacalai Tesque, Cat $\sharp$ 08458-16) supplemented with $10 \%$ FBS (Sigma) and Penicillin-Streptomycin (Nacalai Tesque). Cells were treated with BMH-21 (Selleckchem, Cat $\sharp$ S7718) for 3 hours. To visualize FCs and DFCs in immunofluorescence, the antibodies to UBF (Santacruz, F9, sc-13125, mouse monoclonal antibody, 1:50 dilution) and FBL (Santacruz, H-140, sc-25397, rabbit polyclonal antibody, 1:50 dilution) were used, respectively. Cells were grown on coverslips (Matsunami; $18 \mathrm{~mm}$ round) and fixed with $4 \%$ paraformaldehyde/PBS at the room temperature for $10 \mathrm{~min}$. Then, the cells were washed three times with 1 $\mathrm{x}$ PBS, permeabilized with $0.5 \%$ Triton-X100/PBS at room temperature for $5 \mathrm{~min}$, and washed three times with 1 x PBS. The cells were incubated with $1 \mathrm{x}$ blocking solution (Roche, Blocking reagent and TBST $[1 \mathrm{x}$ TBS containing $0.1 \%$ Tween 20]) at room temperature for 1 hour. Then, the coverslips were incubated with primary antibodies in $1 \mathrm{x}$ blocking solution at room temperature for 1 hour, washed three times with TBST for $5 \mathrm{~min}$, incubated with secondary antibodies at room temperature for 1 hour, and washed three times with TBST for 5 min. The coverslips were mounted with Vectashiled hard set mounting medium with DAPI (Vector, H-1500). Superresolution images were acquired using ZEISS LSM900 with Airyscan 2. Quantification of FCs marked by UBF staining within the nucleoli was performed using Volocity 6.3 software (PerkinElmer) with an intensity threshold and a separate touching objects processing.

\section{RESULTS}

\section{Lateral osmotic pressure in DFC layer is enhanced by condensation of RBPs}

We first treat simple cases in which the curvature of the interface between FC and DFC is negligible, $\left(r_{\mathrm{ex}}-\right.$ $\left.r_{\text {in }}\right) / r_{\text {in }} \ll 1$, see Figure 3 . In such cases, the volume fractions of nascent RNA units $\phi_{\mathrm{r}}$ and RBPs $\phi_{\mathrm{p}}$ are uniform in the DFC layer. The volume fractions are derived by using eqs. (12) - (14), see Figure 4. Nascent pre-rRNA transcripts are occupied by RBPs for cases in which the binding energy $\epsilon$ is large. RBPs are attracted to the RBPs bound to the nascent pre-rRNA transcripts due to the attractive interaction between RBPs, where the magnitude of the interaction is proportional to the interaction parameter $\chi$.

For cases in which the interaction parameter $\chi$ and the surface density of nascent RNA transcripts are small, the 


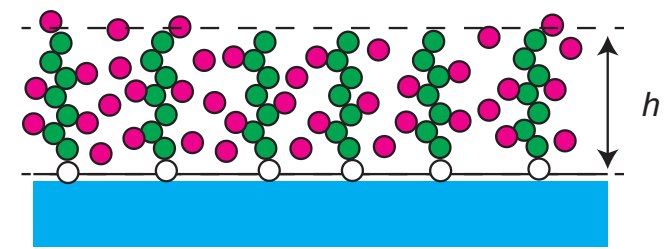

Figure 3. Nascent pre-rRNA transcripts at a planer surface, $\left(r_{\mathrm{ex}}-\right.$ $\left.r_{\text {in }}\right) / r_{\text {in }} \ll 1$. The volume fractions of nascent RNA units, freely diffusing RBPs, RBPs bound to nascent RNA transcripts, and solvent molecules are uniform in the DFC layer.

a.

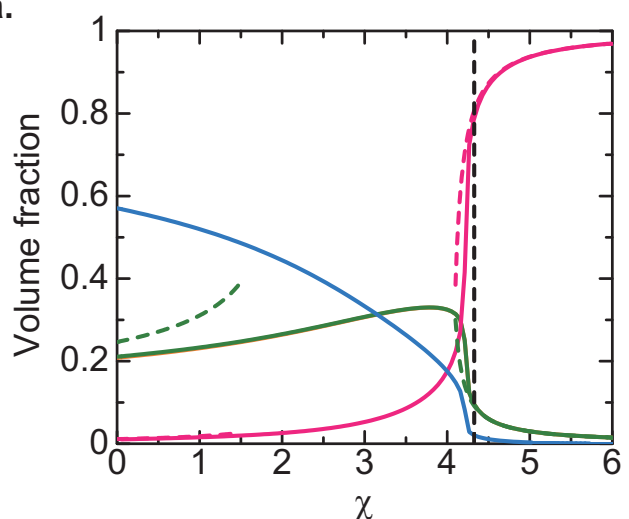

b.

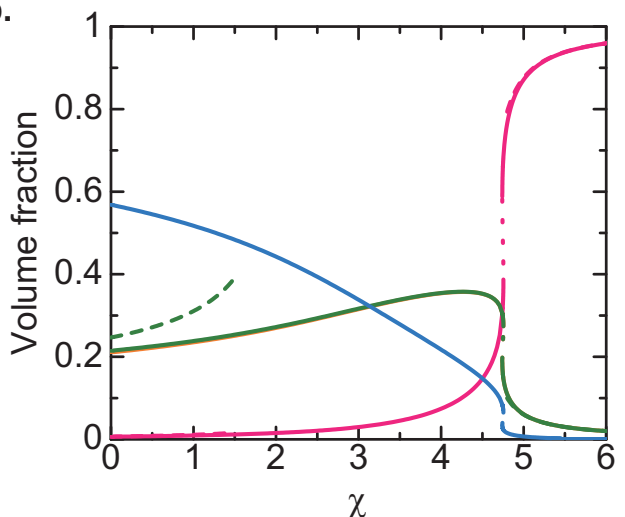

Figure 4. The volume fractions of nascent RNA units (green), freely diffusing RBPs (magenta), RBPs bound to RNA units (orange), and solvent molecules (cyan) in a DFC layer vs the interaction paramter $\chi$ for $\mu_{\mathrm{p}} /\left(k_{\mathrm{B}} T\right)=-4.0$ (a) and -4.5 (b). The solid lines are stable solutions and the dotted lines are unstable solutions. The black broken line indicates the interaction parameter at the critical point $\left(\chi_{\mathrm{c}}=-4.3\right)$. The broken lines are derived by using the approximate solutions, eqs. (15) and (16), for the small and large values of $\chi$, respectively. We used $\epsilon=8.0, \sigma_{0} b^{2}=0.1$, and $\Pi b^{3} /\left(k_{\mathrm{B}} T\right)=0.0$ for the calculations. The orange line mostly overlaps with the green line because of the large binding energy used in the calculation.

major component of the DFC layer is solvent, see the cyan line in Figure $4 \mathbf{a}$. For $\chi<2$ (and $\Pi \approx 0$ ), the volume fraction $\phi_{\mathrm{r}}$ of nascent RNA units has an asymptotic form

$\phi_{\mathrm{r}}=\left(\frac{3 \sigma^{2} b^{4}}{2-\chi}\right)^{1 / 3}$,

which is derived by expanding eq. (13) in a power series of $\phi_{\mathrm{r}}$ for $\phi_{\mathrm{p}} \rightarrow 0$, assuming that the binding energy $\epsilon$ is large enough to ensure $\sigma_{\mathrm{p}}=1$ with this limit, see sec. S3.1 in the
Supplementary File. Eq. (15) is indeed the form of the polymer volume fraction in the Alexander brush $(20,21)$, see the green broken line in Figure $4 \mathbf{a}$ and sec. S1 in the Supplementary Files. This results from the fact that the interaction between RBPs bound to nascent RNA units is repulsive for $\chi<2$ when the major component of the DFC layer is solvent. The repulsive interaction between RBPs bound to nascent rRNA units becomes weaker as the interaction parameter $\chi$ increases for $\chi<2$ and the interaction eventually becomes attractive for $\chi>2$. The volume fraction $\phi_{\mathrm{r}}$ of nascent RNA increases with increasing the interaction parameter $\chi$ for $2<\chi<-\mu_{\mathrm{p}} /\left(k_{\mathrm{B}} T\right)$. It is because nascent RNA transcripts collapse due to the attractive interactions between RBPs bound to nascent RNA transcripts.

The volume fraction $\phi_{\mathrm{p}}$ of RBPs in the DFC layer increases steeply with increasing the interaction parameter $\chi$ at the proximity of the critical value of the interaction parameter $\chi_{\mathrm{c}}$ and then asymptotically approaches to unity, see the magenta line in Figure $4 \mathbf{a}$. For $\chi>-\mu_{\mathrm{p}} /\left(k_{\mathrm{B}} T\right)$, the volume fraction $\phi_{\mathrm{r}}$ of nascent RNA transcripts has the form

$$
\phi_{\mathrm{r}}=\frac{3 \sigma^{2} b^{4}}{\chi+\mu_{\mathrm{p}} /\left(k_{\mathrm{B}} T\right)}
$$

with $\Pi \approx 0$, see the green broken line in Figure $4 \mathbf{a}$. We derived eq. (16) by using the fact that the volume fraction of solvent molecules, $\phi_{\mathrm{s}}\left(=1-\phi_{\mathrm{p}}-\left(1+\sigma_{\mathrm{p}}\right) \phi_{\mathrm{r}}\right)$, is small, see sec. S3.1 in the Supplementary File. For $\chi>-\mu_{\mathrm{p}} /\left(k_{\mathrm{B}} T\right)$, the volume fraction $\phi_{\mathrm{r}}$ of nascent RNA units decreases with increasing the interaction parameter $\chi$, implying that nascent RNA transcripts are stretched as the interaction parameter $\chi$ increases. The stretching of nascent RNA transcripts is expected because the solvent is replaced by RBPs diffusing freely in the DFC layer; RBPs bound to nascent RNA units can access to RBPs without reaching to other nascent RNA units and the mixing entropy of freely diffusing RBPs gives rise to the effective repulsive interaction between the complexes $(24,25)$. Indeed, the primary contribution to the repulsive interaction is the mixing entropy of solvent, see the second term of eq. (13).

The chemical potential $\mu_{\mathrm{p}}$ is determined by the concentration of the RBPs in the nucleosol. For cases in which the chemical potential $\mu_{\mathrm{p}}$ is larger than the critical value $\mu_{\mathrm{pc}}$, the volume fractions, $\phi_{\mathrm{p}}$ and $\phi_{\mathrm{r}}$, of RBPs and nascent RNA units are continuous function of the interaction parameter $\chi$, see Figure 4a. For cases in which the chemical potential is smaller than the critical value $\mu_{\mathrm{pc}}$, the volume fractions, $\phi_{\mathrm{p}}$ and $\phi_{\mathrm{r}}$, jump at the threshold value $\chi_{\mathrm{tr}}$ of the interaction parameter, see Figure $4 \mathbf{b}$. In the limit of small volume fraction of nascent RNA transcripts, $\phi_{\mathrm{r}} \rightarrow 0$ and $\sigma \rightarrow 0$, our model returns to the simple mean field theory of the binary mixture of RBPs and solvent molecules (23). The latter theory predicts that the phase separation of the binary mixture happens for $\chi=\chi_{\mathrm{tr}}$, where the interaction parameter at the threshold is $\chi_{\mathrm{tr} 0}=-\mu_{\mathrm{p}} /\left(k_{\mathrm{B}} T\right)$, see the broken line in Figure 5. FBL molecules show phase separation in vitro with the physiological concentration, implying that $\mu_{\mathrm{p}}>-\chi k_{\mathrm{B}} T$ (15). Our theory predicts that the threshold value $\chi_{\mathrm{tr}}$ of the interaction parameter in the DFC layer is somewhat larger than the threshold value of the binary 


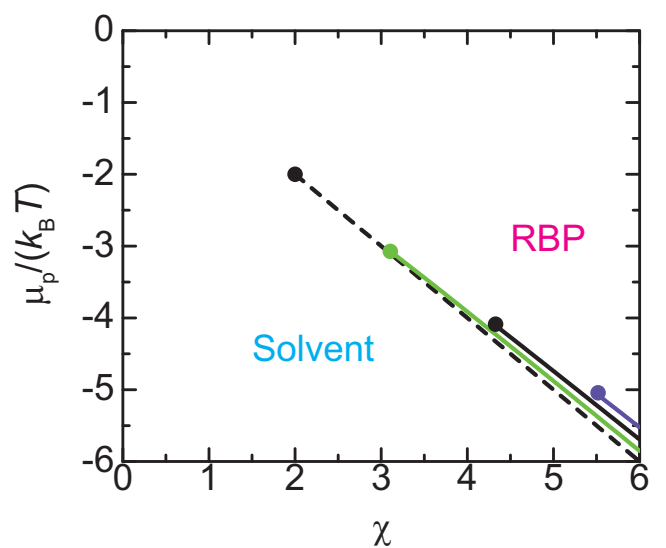

Figure 5. Phase diagram of a DFC layer with respect to the chemical potential $\mu_{\mathrm{p}}$ and the interaction parameter $\chi$. The solid lines are the threshold interaction parameter $\chi_{\operatorname{tr}}$ for $\sigma b^{2}=0.05$ (light green), 0.1 (black), and 0.15 (purple). The DFC layer is rich in solvent molecules for $\chi<\chi_{\operatorname{tr}}$ and in RBPs for $\chi>\chi_{\mathrm{tr}}$. The threshold interaction parameter $\chi_{\mathrm{tr}}$ for the binary mixture of RBPs and solvent molecules is shown by the black broken line. We used $\epsilon=8.0$ and $\Pi b^{3} /\left(k_{\mathrm{B}} T\right)=0.0$ for the calculations.

mixture because nascent RNA transcripts in the layer act as impurity, see Figure 5.

Nascent RNA transcripts generate osmotic pressure $\Pi_{\|}$in the lateral direction (parallel to the DFC layer). The lateral osmotic pressure counteracts the surface tension. The lateral osmotic pressure $\Pi_{\|}$has the form

$$
\begin{aligned}
\frac{\phi_{\mathrm{r}}}{\sigma N_{\mathrm{r}}} \frac{\Pi_{\|}}{k_{\mathrm{B}} T}= & -\log \left(1-\phi_{\mathrm{p}}-\left(1+\sigma_{\mathrm{p}}\right) \phi_{\mathrm{r}}\right)-\left(1+\sigma_{\mathrm{p}}\right) \phi_{\mathrm{r}} \\
& -\chi\left(\phi_{\mathrm{p}}+\sigma_{\mathrm{p}} \phi_{\mathrm{r}}\right)^{2}-\frac{\Pi b^{3}}{k_{\mathrm{B}} T}
\end{aligned}
$$

for cases in which the curvature of the surface of the microphase is small. Eq. (17) is derived by using the thermodynamic relationship $\Pi_{\|} b^{3}=\sigma^{2} \frac{\partial}{\partial \sigma}\left(\frac{f}{\sigma}\right)$, where $f$ is the integrand of eq. (3). The osmotic pressure in the DFC layer is anisotropic due to the (entropic) elasticity of nascent RNA transcripts (it is shown by substituting eq. (13) into eq. (17)) (23). The lateral osmotic pressure is positive even for $2<$ $\chi<\chi_{\mathrm{tr}}$, where nascent RNA units show attractive interactions, see Figure 6. It is because nascent RNA transcripts already collapse to the optimal volume fraction; the deviation from the optimal volume fraction always increases the free energy.

The lateral osmotic pressure decreases with increasing the interaction parameter $\chi$ for cases in which the parameter $\chi$ is relatively small, see Figure 6 . For $\chi<2$, the lateral osmotic pressure has an asymptotic form

$$
\frac{\Pi_{\|} b^{2}}{N_{\mathrm{r}} k_{\mathrm{B}} T}=3^{1 / 3}\left(\sigma b^{2}\right)^{7 / 3}(2-\chi)^{2 / 3},
$$

see the broken lines in Figure 6. Eq. (18) is derived by using eq. (15), see eqs. (13) and (17). In contrast, the lateral osmotic pressure increases steeply with increasing the interaction parameter $\chi$ for cases in which the parameter $\chi$ is large, see Figure 6, reflecting the stretching of nascent RNA transcripts, see the green line in Figure 4. For $\chi>-\mu_{\mathrm{p}} /\left(k_{\mathrm{B}} T\right)$, the lateral

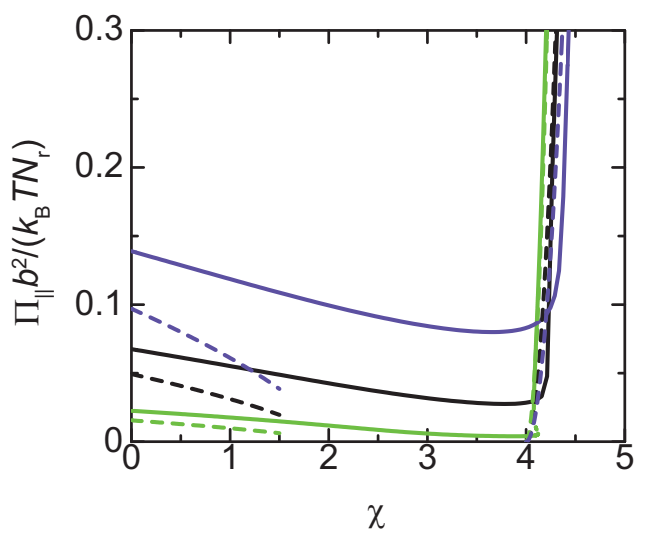

Figure 6. Lateral osmotic pressure $\Pi_{\|}$vs the interaction parameter $\chi$ for $\sigma b^{2}=0.05$ (light green), 0.1 (black), and 0.15 (purple). The solid lines are stable solutions and the dotted lines are unstable solutions. The broken lines are derived by using the asymptotic forms, eqs. (18) and (19), for small and large values of the interaction parameter $\chi$. We used $\epsilon=8.0$ and $\Pi b^{3} /\left(k_{\mathrm{B}} T\right)=0.0$ for the calculations.

osmotic pressure has an asymptotic form

$$
\frac{\Pi_{\|} b^{2}}{N_{\mathrm{r}} k_{\mathrm{B}} T}=\frac{1}{3 \sigma b^{2}}\left(\chi+\frac{\mu_{\mathrm{p}}}{k_{\mathrm{B}} T}\right)^{2},
$$

see the broken curves in Figure 6. Eq. (19) is derived by using eq. (16), see also eqs. (13) and (17). Our theory therefore predicts that the lateral osmotic pressure is enhanced by the condensation of RBPs in the DFC layer. In the following, we treat the regime of large interaction parameter, $\chi>-\mu_{\mathrm{p}} /\left(k_{\mathrm{B}} T\right)$, see Figure 5 .

The volume fraction $\phi_{\mathrm{r}}$ of nascent RNA units increases with increasing the surface density $\sigma$ of nascent RNA transcripts and eventually becomes larger than the volume fraction $\phi_{\mathrm{p}}$ of freely diffusing RBPs, see the green and magenta lines in Figure 7a. In the limit of large surface density $\sigma$, the volume fraction $\phi_{\mathrm{r}}$ of nascent RNA units has an asymptotic form

$\phi_{\mathrm{r}}=\frac{1}{2}-\frac{1}{2}\left(1+\mathrm{e}^{-\frac{\mu_{\mathrm{p}}}{k_{\mathrm{B}} T}+\chi}\right) \mathrm{e}^{-1-\frac{\chi}{4}-6 \sigma^{2} b^{4}}$,

see the green broken line in Figure 7a. Eq. (20) is derived by expanding eqs. (12) and (13) with respect to the volume fractions $\phi_{\mathrm{p}}$ and $\phi_{\mathrm{s}}\left(\equiv 1-\phi_{\mathrm{p}}-2 \phi_{\mathrm{r}}\right)$, see also sec. S3.2 in the Supplementary File. The volume fraction $\phi_{\mathrm{r}}$ of nascent RNA units is determined by the balance of the attractive interactions between RBPs and the elasticity of nascent RNA transcripts. The lateral osmotic pressure decreases with increasing the surface density $\sigma$ of nascent RNA transcripts for small values of the surface density $\sigma$, see eq. (19) and Figure 7b. In contrast, the lateral osmotic pressure increases with increasing the surface density $\sigma$ for large values of the surface density $\sigma$, see Figure 7. In the limit of large surface density $\sigma$, the lateral osmotic pressure has the form

$$
\frac{\Pi_{\|} b^{2}}{k_{\mathrm{B}} T N_{\mathrm{r}}}=12 \sigma^{3} b^{6},
$$

see the broken line in Figure $7 \mathbf{b}$. 
a.

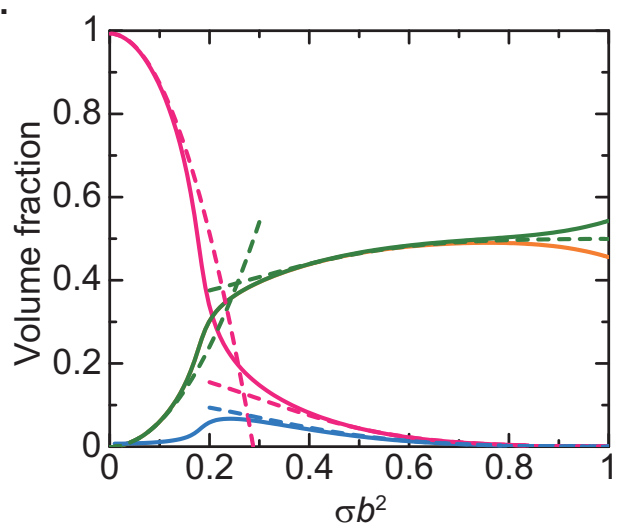

b.

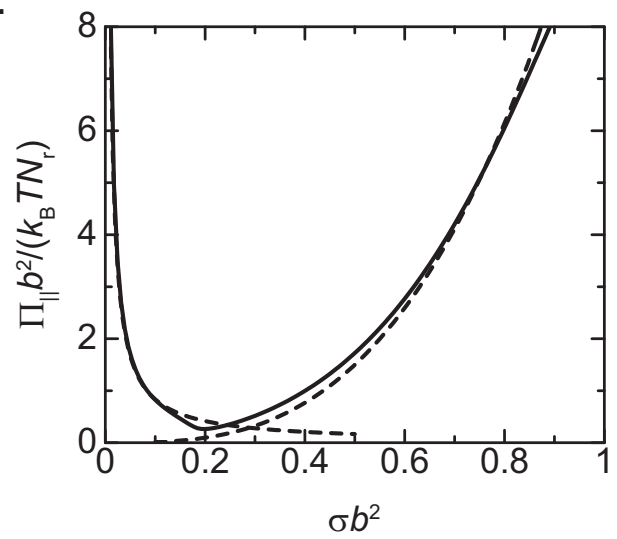

Figure 7. The composition (a) and the lateral osmotic pressure (b) in a DFC layer vs the surface density $\sigma$ of nascent RNA transcripts. The volume fractions of nascent RNA units (green), freely diffusing RBPs (magenta), RBPs bound to RNA units (orange), and solvent molecules (cyan) in a DFC layer are shown. The broken lines in a are derived by using the asymptotic forms, eqs. (16) and (20). The broken lines in $\mathbf{b}$ are derived by using eqs. (19) and (21). We used $\epsilon=8.0, \chi=4.5, \mu_{\mathrm{p}} /\left(k_{\mathrm{B}} T\right)=-4.0$ and $\Pi b^{3} /\left(k_{\mathrm{B}} T\right)=$ 0.0 for the calculations. The orange line in a mostly overlaps with the green line because of the large binding energy used in the calculation.

\section{Nascent RNA transcripts suppress the growth of microphases}

For cases in which the curvature of the surface of the microphase is not negligible, the volume fraction $\phi_{\mathrm{r}}$ of nascent RNA units depends on the distance $r$ from the center of the microphase, see Figure 8. The thickness of the DFC layer depends on the number $N_{\mathrm{r}}$ of units, see eq. (4), but the local volume fraction $\phi_{\mathrm{r}}$ does not, see eqs. (12) and (13). The volume fraction $\phi_{\mathrm{r}}$ of nascent RNA units decreases with increasing the distance $r$ from the center of the microphase because of the (entropic) elasticity of nascent RNA transcripts, see Figure 8.

For small surface density $\sigma$ of nascent RNA transcripts, the volume fraction $\phi_{\mathrm{r}}$ has an asymptotic

$\phi_{\mathrm{r}}=\frac{3 \sigma^{2} b^{4}}{\chi+\mu_{\mathrm{p}} /\left(k_{\mathrm{B}} T\right)} \frac{r_{\mathrm{in}}^{4}}{r^{4}}$,

see the green broken line in Figure 8a. Eq. (22) is derived in a similar manner to eq. (16), see also sec. S3.1 in the Supplementary File. For large surface density $\sigma$ of nascent a.

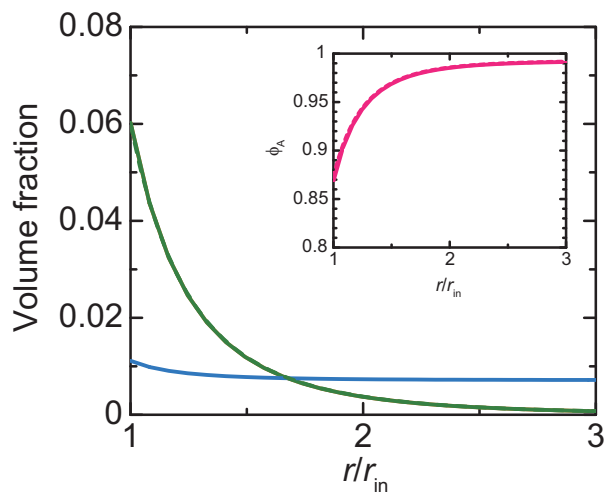

b.

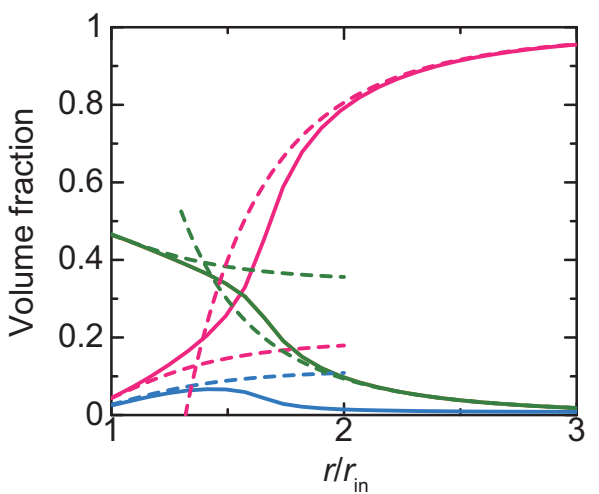

Figure 8. Profile of the volume fractions of nascent RNA units (green), freely diffusing RBPs (magenta), RBPs bound to nascent RNA (orange), and solvent molecules (cyan) for $\sigma b^{2}=0.1$ (a) and 0.5 (b). The broken lines in $\mathbf{a}$ is derived by using eq. (22). The broken line in $\mathbf{b}$ is derived by using eq. (23) for $r / r_{\text {in }} \approx 1$ and by using eq. (22.) for $r / r_{\text {in }} \gg 1$. We used $\epsilon=8.0, \chi=4.5$, $\mu_{\mathrm{p}} /\left(k_{\mathrm{B}} T\right)=-4.0$ and $\Pi b^{3} /\left(k_{\mathrm{B}} T\right)=0.0$ for the calculations. The orange line mostly overlaps with the green line because of the large binding energy used in the calculation.

RNA transcripts, the volume fraction $\phi_{\mathrm{r}}$ has an approximate form

$\phi_{\mathrm{r}}=\frac{1}{2}-\frac{1}{2}\left(1+\mathrm{e}^{\frac{\mu_{\mathrm{p}}}{k_{\mathrm{B}} T}+\chi}\right) \mathrm{e}^{-1-\frac{\chi}{4}-\frac{6 \sigma^{2} b^{4} r_{\text {in }}^{4}}{r^{4}}}$.

Eq. (23) is derived in a similar manner to eq. (20), see also sec. 3.2 in the Supplementary File. Eq. (23) is effective for $r / r_{\text {in }} \approx 1$, where the volume fraction $\phi_{\mathrm{r}}$ of nascent RNA units is relatively large. Eq. (22) is effective for $r / r_{\text {in }} \gg 1$, where the volume fraction $\phi_{\mathrm{p}}$ of freely diffusion RBPs is relatively large.

Now we analyze the free energy as a function of the radius $r_{\text {in }}$ of the microphase by substituting the volume fractions, $\phi_{\mathrm{r}}$ and $\phi_{\mathrm{p}}$, of nascent RNA units and RBPs into eq. (9). Because the surface density $\sigma$ of nascent RNA transcripts is proportional to the radius $r_{\text {in }}$ of the microphase, see eq. (2), we treat the surface density $\sigma$ as the rescaled radius $r_{\text {in }}$. The surface free energy, the second and third terms of in the square bracket of eq. (9), decreases monotonically with increasing the radius $r_{\text {in }}$ of the microphase, see the light green curve in Figure 9. This implies that when the transcription is suppressed, the size of the microphase increases as much as the components of FC are available to minimize the surface 


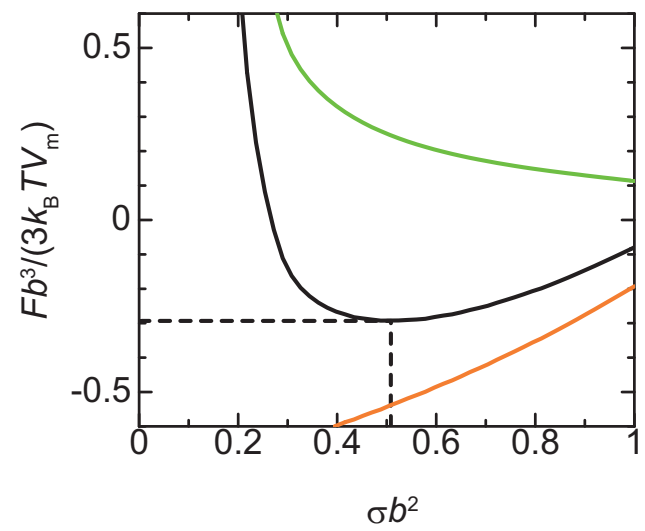

Figure 9. Free energy $F$ vs radius $r_{\text {in }}$ of microphases (black). The radius $r_{\text {in }}$ of microphases is proportional to the surface density $\sigma$ of nascent RNA transcripts, see eq. (2). The surface free energy (light green) and the free energy in the DFC layer (orange) are shown separately. We used $\epsilon=8.0, \chi=$ $4.5, \mu_{\mathrm{p}} /\left(k_{\mathrm{B}} T\right)=-4.0, N_{\mathrm{r}} \sigma b^{3} / r_{\mathrm{in}}=0.1, \gamma_{\mathrm{p}} \sigma b^{3} /\left(k_{\mathrm{B}} T r_{\mathrm{in}}\right)=0.1$, and $\Pi b^{3} /\left(k_{\mathrm{B}} T\right)=0.0$ for the calculations.

free energy. In contrast, the free energy of the DFC layer, the first term in the square bracket of eq. (9), increases with increasing the radius $r_{\text {in }}$ of the microphase. The free energy thus has a minimum due to the balance of these free energy contributions, see the black line in Figure 9. Our theory predicts that the size of microphases increases to the radius at the minimum of the free energy and the growth is suppressed by the free energy of the DFC layer.

\section{Radius of microphases decreases with increasing transcription rate and time scale of RNA processing}

Because the growth of microphases is suppressed by the lateral osmotic pressure in the DFC layer, we expect that the radius $r_{\text {in }}$ of microphases at the free energy minimum depends on the number $N_{\mathrm{r}}$ of units in nascent RNA transcripts, the processing time $\tau_{\mathrm{pr}}$, and the transcription rate $k_{\mathrm{tx}}$, where

$k_{\mathrm{tx}} \tau_{\mathrm{pr}}=\frac{\sigma b^{3}}{r_{\mathrm{in}}}$.

The transcription rate $k_{\mathrm{tx}}$ is independent of the radius $r_{\mathrm{in}}$ because the surface density $\sigma$ of nascent RNA transcripts is proportional to the radius $r_{\text {in }}$, see eq. (2).

Our theory predicts that the radius of microphases decreases with increasing the transcription rate $k_{\mathrm{tx}}$ and the processing time $\tau_{\text {pr }}$, see Figure 10 and eq. (25). It is because the surface density $\sigma$ of nascent RNA transcripts increases with increasing the transcription rate $k_{\mathrm{tx}}$ and the processing time $\tau_{\mathrm{pr}}$ and this increases the lateral osmotic pressure that suppresses the growth of microphases. Our theory therefore predicts that the radius of microphases decreases when one upregulate the transcription of pre-rRNA and/or suppresses the processing of pre-rRNA.

The radius $r_{\text {in }}$ decreases with increasing the number $N_{\mathrm{r}}$ of units in each nascent RNA transcript for small $N_{\mathrm{r}}$ and the radius $r_{\text {in }}$ increases with increasing the number $N_{\mathrm{r}}$ of units for large $N_{\mathrm{r}}$, see Figure 11. For small number $N_{\mathrm{r}}$ of units in nascent RNA transcripts, the surface density $\sigma$ of nascent

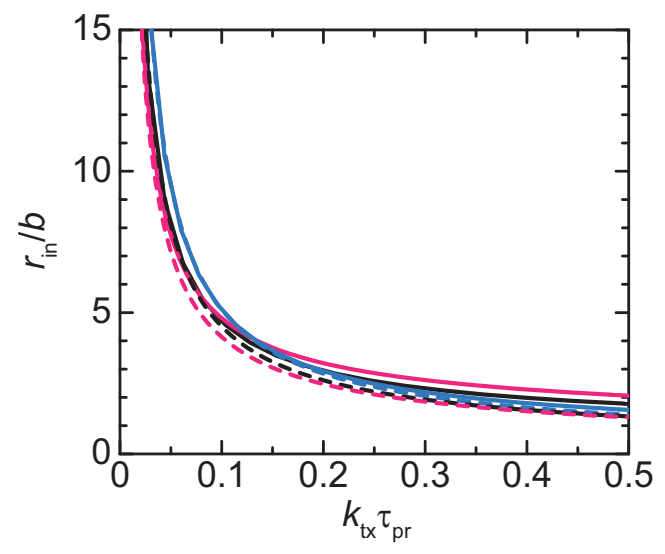

Figure 10. Radius $r_{\text {in }}$ of microphases vs the transcription rate $k_{\mathrm{tx}} \tau_{\mathrm{pr}}$ (defined by eq. (24)). The broken curves are derived by using eq. (25). We used $\epsilon=8.0, \chi=4.5, \mu_{\mathrm{p}} /\left(k_{\mathrm{B}} T\right)=-4.0, \gamma_{\mathrm{p}} b^{2} /\left(k_{\mathrm{B}} T\right)=1.0$, and $\Pi b^{3} /\left(k_{\mathrm{B}} T\right)=0.0$ for the calculations.

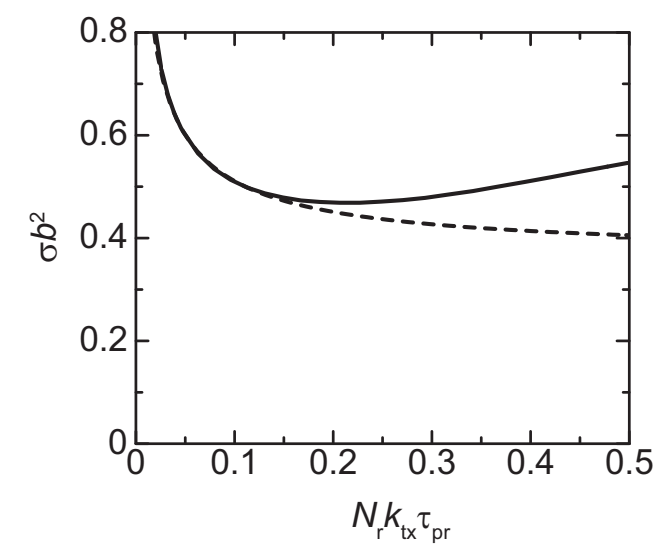

Figure 11. Radius $r_{\text {in }}$ of microphases vs the number $N_{\mathrm{r}}$ of units in a nascent RNA transcript. The radius $r_{\text {in }}$ of microphases is proportional to the surface density $\sigma$ of microphases and thus $k_{\mathrm{tx}}$ defined by eq. (24) is independent of the radius $r_{\mathrm{in}}$, see eq. (2). The broken line is derived by using eq. (25). We used $\epsilon=8.0, \chi=4.5, \mu_{\mathrm{p}} /\left(k_{\mathrm{B}} T\right)=-4.0, \gamma_{\mathrm{p}} \sigma b^{5} /\left(k_{\mathrm{B}} T r_{\mathrm{in}}\right)=0.1$, and $\Pi b^{3} /\left(k_{\mathrm{B}} T\right)=0.0$ for the calculations.

RNA transcripts has an asymptotic form

$\sigma b^{2}=\left(\frac{\gamma_{\mathrm{p}} b^{2}}{12 N_{\mathrm{r}} k_{\mathrm{B}} T}\right)^{1 / 3}\left(1+\frac{6 N_{\mathrm{r}} \sigma b^{3}}{r_{\text {in }}}\right)^{1 / 3}$,

see the broken line in Figure 11. The derivation of eq. (25) is shown in sec. S4.1 in the Supplementary File. The deviation between the asymptotic form, eq. (25), and the numerical calculation results from the fact that the volume fraction of RBPs freely diffusing in the DFC layer dominates the volume fraction of nascent RNA units at the proximity to the interface between DFC and GC, see Figure $8 \mathbf{b}$; this increases the exterior radius $r_{\mathrm{ex}}$ and the surface tension at the interface between DFC and GC.

\section{Mild inhibition of Pol I increases the size of FCs}

Our theoretical model predicts that the size of FCs becomes larger as the number of nascent pre-rRNAs reduces. To experimentally test this effect, we used BMH-21, a specific 
inhibitor of Pol I, which reduces nascent pre-rRNA levels in a dose-dependent manner (26). In untreated HeLa cells, small foci of UBF (a marker for FCs) and FBL (a marker for DFC) proteins were dispersed within the nucleolus (26) (Figure 12a). UBF and FBL proteins were relocalized to nucleolar caps in the presence of high-doses of BMH-21 (0.5 and $1.0 \mu \mathrm{M}$ ), as reported (26) (Figure 12a). Strikingly, medium dose treatment of BMH-21 $(0.0625,0.125$, and $0.25 \mu \mathrm{M})$ caused formation of larger spherical FCs and DFCs compared to those in untreated cells (Figure 12a). We then quantified the size of the FCs under untreated and medium dose treated conditions (Figure 12b-e). The longest axis and area of the FCs increases with increasing BMH-21 dose. These data suggest that mild Pol I inhibition, which causes reduction of nascent pre-rRNAs, increases the size of FCs in consistent with our theoretical prediction.

\section{DISCUSSION}

Our theory predicts that nascent pre-rRNA transcripts at the surfaces of FC microphases in a nucleolus generate the lateral osmotic pressure that suppresses the growth of microphases. The radius of microphases decreases with increasing the transcription rate $k_{\mathrm{tx}}$ and/or with increasing the processing time $\tau_{\text {pr }}$. The prediction that the radius of FCs decreases continuously with increasing the transcription rate is in agreement with our experimental results that the size of FCs increased with decreassing the transcription level of pre-rRNA by BMH-21. The inhibition of the processing factor, such as uL18 (RPL5) and uL5 (RPL11), changes the multiphase structure of nucleoli $(3,27)$, but the quantitative relationship between its effects on the residence time of prerRNA transcripts at the surfaces of FCs and the size of FCs remains to be experimentally determined. In many cases, the multi-valent interaction between complexes of RNA and RBPs drives the growth of condensates $(12,13)$, see Figure $13 \mathbf{a}$. The size of such condensates increases with increasing the transcription rate. In contrast, for the case of FCs in nucleoli, the multi-valent interaction between the complexes of nascent RNA and RBPs rather suppresses the growth of FCs (16). The size of FCs decreases with increasing the transcription rate. Our theory predicts that the suppression of the growth of FCs by the multi-valent interactions between complexes of nascent pre-rRNA transcripts and RBPs results from the fact that these complexes are end-grafted to the surfaces of FCs via Pol I, see Figure $13 \mathbf{b}$. This result is also supported by a very recent experiment on a bioengineered system (28).

We have used a couple of assumptions to simplify the theory:

1. We assumed that FCs are spherical. FCs of arbitrary shape can be studied for cases in which the thickness $h$ of DFC is smaller than the radius $r_{\text {in }}$ of FCs, by using the curvature elastic energy, which is derived by the expansion of the free energy $F_{\mathrm{d}}$ with respect to $h / r_{\text {in }}(23,29)$. However, because only spherical FCs have been observed experimentally, our present treatment is probably enough.

2. We determined the volume fractions, $\phi_{\mathrm{r}}$ and $\phi_{\mathrm{p}}$, of nascent RNA transcripts in the steady state by minimizing the free energy. With this assumption, the chemical potential of RBPs and the osmotic pressure are uniform in DFC layers. The conformation of nascent RNA transcripts is determined by the balance of the relaxation dynamics of nascent RNA transcripts and the kinetics of adding NTPs to the transcripts. With this dynamics, the local concetration of nascent RNA units near the surfaces of FCs increases, analogous to the dynamics of DNA at the surface of transcriptional condensates (30). The dynamics of nascent RNA transcripts may be worth to study in the future research.

3. We used a Flory-type mean field theory to treat nascent RNA transcripts at the surfaces of FCs. The advantage of using the mean field theory is that one can use the same free energy throughout the theory and greatly simplifies the discussion. In a more precise treatment, the free energy of polymers is estimated as the thermal energy $k_{\mathrm{B}} T$ per blob (25). With this free energy, the surface density $\sigma b^{2}$ at the minimum of the free energy is derived in the form

$\sigma b^{2}=c_{0}\left(\frac{\gamma_{\mathrm{p}} b^{2}}{3 N_{\mathrm{r}} k_{\mathrm{B}} T}\right)^{2 / 5}\left(1+\frac{5 N_{\mathrm{r}} \sigma b^{3}}{r_{\mathrm{in}}}\right)^{2 / 5}$

where $c_{0}$ is the numerical factor of order unity. The derivation of eq. (26) is shown in sec. S4.2 in the Supplementary File. The exponents and numerical factors in eq. (26) are somewhat different from the corresponding form, eq. (25), of the mean field theory. However, both approximations demonstrate the same physics.

4. The number of transcriptionally active rDNA repeats in a nucleolus does not depend on the number and size of FCs. Recent experiments have shown that the half of the coding regions of rDNA are not methylated and are probably transcriptionally active, whereas the other coding regions are methylated and are probably transcriptionally inactive (31). It is consistent with the previous experiment that showed that only half of coding rDNA units are transcriptionally active (18). These experimental results imply that the number of active rDNA repeats are already determined by the DNA methylation. More experimental inputs are necessary to treat the transition of rDNA coding units between transcriptionally active and inactive states.

5. We assumed that all FCs in a nucleolus have equal size. Indeed, the production of pre-rRNA transcripts at the surfaces of FCs creates the gradient of the osmotic pressure (32). The size of a FC thus depends on the position of the FC in the nucleolus. We envisage a future research to elucidate the relationship beween the size of FCs and the fluxes of ribosomal proteins and pre-ribosomal RNA.

6. RBPs diffuse in the DFC layer when they are not bound to nascent RNA transcrits. Recent experiments has shown that the transcription of pre-rRNA is suppressed when the DFC layer is solidified (33). 
a.

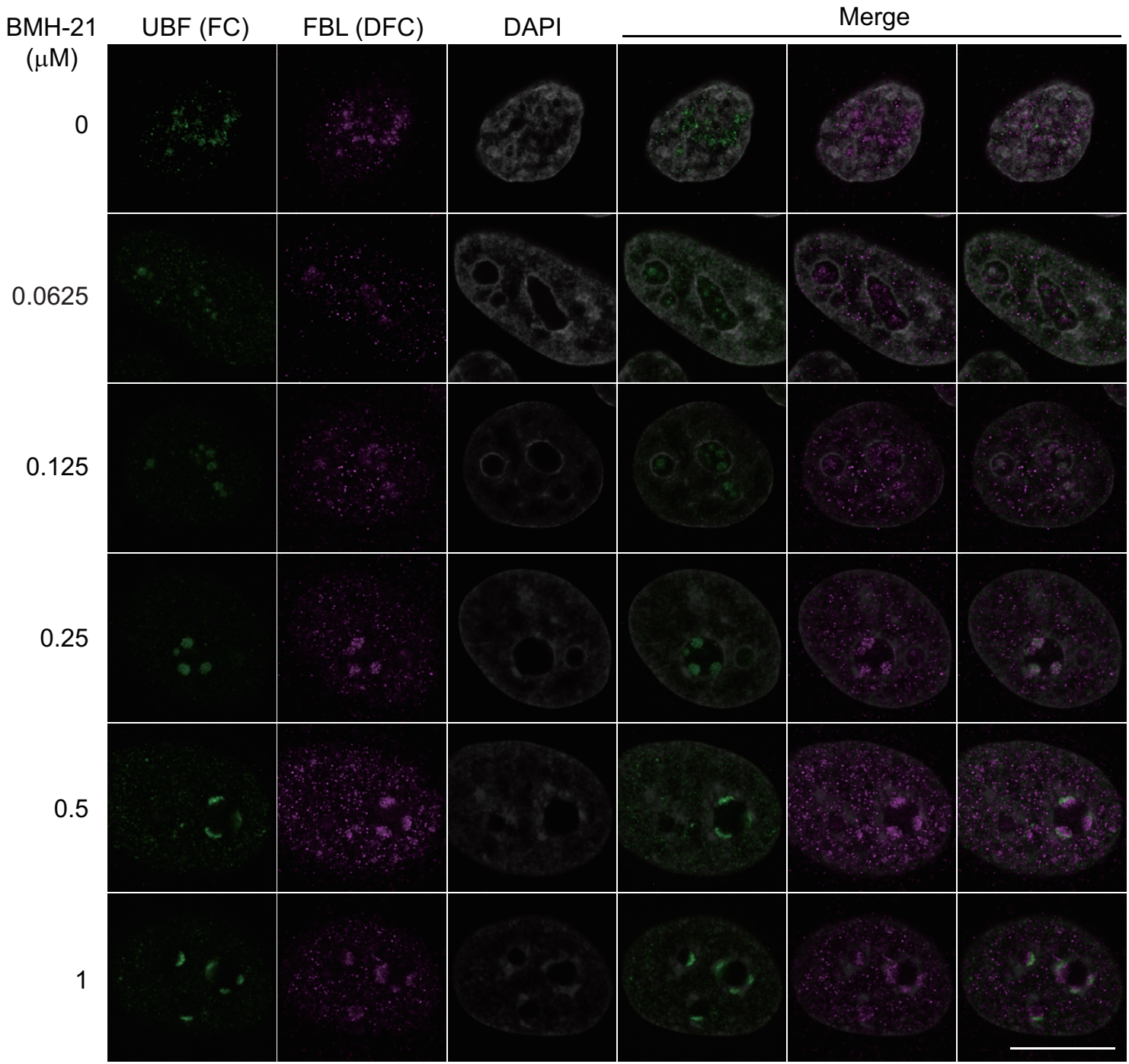

b.

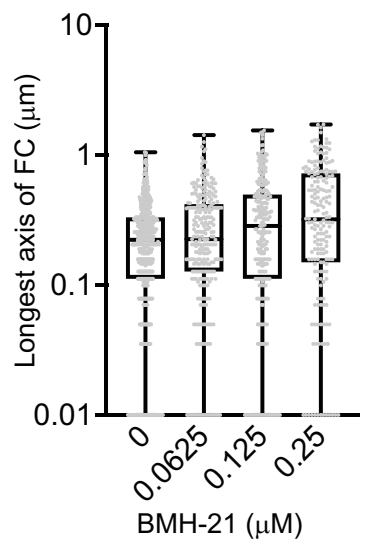

c.

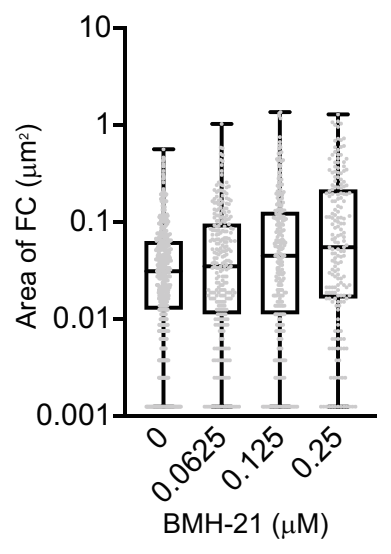

d.

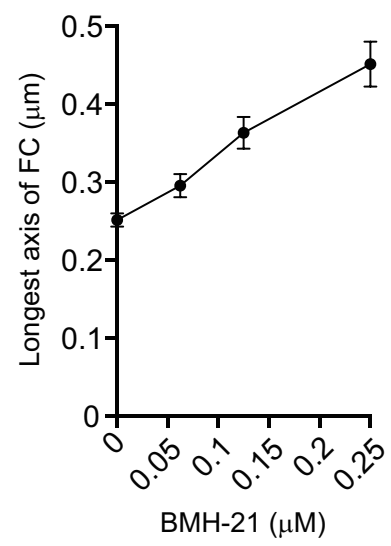

e.

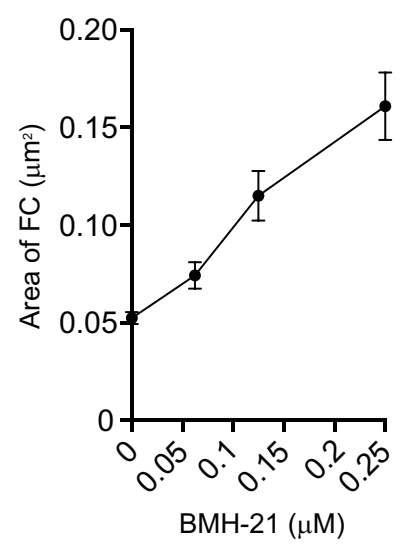

Figure 12. Mild RNA polymerase I inhibition increases the size of FCs. a. Immunofluorescence of UBF (FC) and FBL (DFC) in BMH-21 treated HeLa cells. Scale bar, $10 \mu \mathbf{m}$. b. and $\mathbf{c}$. Quantification of longest axis $(\mathbf{b})$ and area $(\mathbf{c})$ of the FCs in cells under indicated conditions. Each box plot shows the median (inside line), 25-75 percentiles (box bottom to top), and 0-100 percentiles (whisker bottom to top). Dots indicate all points of quantified data (BMH-21: $0 \mu \mathrm{M}, \mathrm{n}=513$; $0.0625 \mu \mathrm{M}, \mathrm{n}=265 ; 0.125 \mu \mathrm{M}, \mathrm{n}=247 ; 0.25 \mu \mathrm{M}, \mathrm{n}=187)$. Mean longest axis and area of the FCs were shown below the graphs. d. and e. Graphs showing changes of mean longest axis (d) and area (e) of the FCs with SEM according to the BMH-21 treatments. 
a.

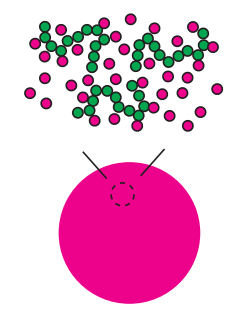

Condensate growth enhanced b.

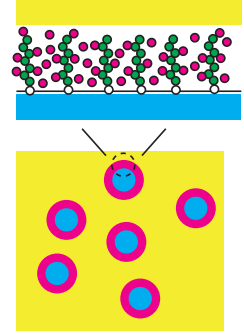

Condensate growth suppressed

Figure 13. Complexes of RNA transcripts and RBPs enhance or suppress the growth of condensates depending on whether the complexes are localized at the interior or the surfaces of the condensates. a. The multi-valent interactions between the complexes of RNA transcripts and RNA-binding proteins (RBPs) enhance the growth of the condensates when these complexes diffuse freely in the condensates. b. The multi-valent interactions between the complexes of RNA transcripts and RBPs suppress the growth of the condensates when these complexes are end-grafted to the surfaces of these condensates.

7. Nascent RNA transcripts are distributed uniformly at the surface of a FC. Indeed, super-resolution microscopy experiments have shown that the DFC layer is indeed the mosic of domains rich in FBL and domains absent of FBL (18). The mechanism of the formation of the mosaic structure is not clear and will be studied in the future research.

Our present theory forms the basis to theoretically study these missing pieces and to understand the detailed mechanism of the assembly and function of the multi-phase structure of nucleolus.

Nascent pre-rRNAs form a hydrophobic DFC layer, which is sandwiched between two relatively hydrophilic GC and FC phases, by making complexes with RBPs, such as FBL. This structure may be somewhat analogous to lipid vesicles in an aquaous solution $(23,29)$. The growth of transcriptional condensates $(8,34)$ and nuclear speckles (35) is also suppressed by nascent RNA transcripts, which are probably localized at their surfaces. Hilbert and coworkers proposed that nascent RNA transcripts act as 'surfactants' at the surfaces of transcriptional condensate because these transcripts are connected with DNA via RNA polymerase II (8). However, this mechanism is effective only at the surfaces between DNA-rich phase and RNA-rich phase, but not to the surfaces between the FC and GC phases in nucleolus, where DNA is a minor component in both of the phases. We have recently shown that the growth of paraspeckles, which are composed of NEAT1_2 architectural RNA transcripts, is suppressed by the excluded volume interactions between the terminal regions of NEAT1_2 $(36,37)$. The size of paraspeckles increases with the upregulation of the transcription of NEAT1_2 $(36,39)$, which is indeed opposite to the response of FCs in nucleoli to the transcription upregulation. The multi-phase structure was also observed in nuclear stress bodies (nSBs) by electron microscopy (40). nSBs are assembled during the thermal stress condition and are composed of HSATIII architectrual RNA and specific RBPs (41). HSATIII is produced by the transcription of the pericentromeric regions, which are enriched with tandem repeats $(42,43)$. These features motivate us to think of a general mechanism involved in the assembly

of the multiple microphases in nuclear bodies. As it has been outlined in this paragraph, transcription and RNA processing play an important role in the assembly of nuclear bodies. It results from the fact that nuclear bodies are scaffolded by architectural RNA.

\section{DATA AVAILABILITY}

The Mathematica file (nucleolusFCprojectVer14.nb) used to derive the data that support the findings of this study are available in figshare with the identifier (https://doi.org/10.6084/m9.figshare.16599446).

\section{SUPPLEMENTARY DATA}

Supplementary Data are available at NAR Online.

\section{FUNDING}

This research was supported by KAKENHI grants from the Ministry of Education, Culture, Sports, Science, and Technology (MEXT) of Japan [to T. Yamamoto (20H05934, 21K03479, 21H00241), T. Yamazaki (19K06479, 19H05250, 21H0025), KN (19K06478), and TH (20H00448, 20H05377)], JST, PRESTO Grant Number JPMJPR18KA (to T. Yamamoto), the Mochida Memorial Foundation for Medical and Pharmaceutical Research (to T. Yamazaki), the Naito Foundation (to T. Yamazaki), the Takeda Science Foundation (to T. Yamazaki), and JST CREST Grant Number JPMJCR20E6 (to T.H.).

\section{CONFLICT OF INTEREST}

None declared.

\section{ACKNOWLEDGEMENTS}

T. Yamamoto acknowledges the fruitful discussion with Noriko Saito (Japanese Foundation for Cancer Research), Yuma Ito (Tokyo Institute of Technology), Satoru Ide (National Institute of Genetics), Yutetsu Kuruma (JAMSTEC), Shintaro Iwasaki (Univ. of Tokyo), Sumio Sugano (Chiba University), Takehiko Kobayashi (Univ. of Tokyo), Haruhiko Siomi (Keio Univ.), and Hideaki Matsubayashi (Johns Hopkins Univ.).

Conflict of interest statement. None declared. 
bioRxiv preprint doi: https://doi.org/10.1101/2021.09.09.459702; this version posted September 10, 2021. The copyright holder for this

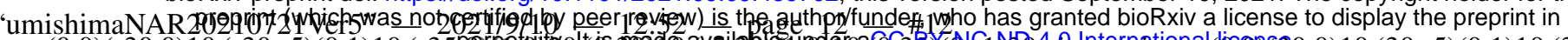

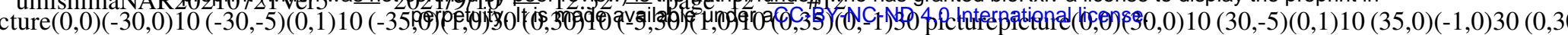

12 Nucleic Acids Research, YYYY, Vol. xx, No. $x x$

\section{REFERENCES}

1. Klinge,S. and Woolford Jr, J.L. (2019) Ribosome assembly coming into focus. Nat. Rev. Mol. Cell Biol., 20, 116-131.

2. McStay,B. and Grummt,I. (2008) The Epigenetics of rRNA Genes: From Molecular to Chromosome Biology. Annu. Rev. Cell Dev. Biol., 24, 131157.

3. Lafontaine,D.L.J., Riback,J.A., Bascetin,R., and Brangwynne,C.P. (2021) The nucleolus as a mutiphase liquid condensate. Nat. Rev. Mol. Cell Biol., 22, 165-182.

4. Berry,J., Weber,S.C., Vaidya,N., Haataja, M. and Brangwynne,C.P. (2015) RNA transcription modulates phase transition driven nuclear body assembly. Proc. Natl. Acad. Sci. USA, 112, E5237-E5245.

5. Nozaki,T., Imai,R., Tanbo,M., Nagashima,R., Tamura,S., Tani,T., Joti,Y., Tomita,M, Hibino,K., Kanemaki,M.T., Wendt,K.S., Okada,Y., Nagai,T. and Maeshima,K. (2017) Dynamic Organization of Chromatin Domains Revealed by Super-Resolution Live-Cell Imaging. Mol. Cell, 67, 282293.

6. Sabari,B.R., Dall'Agnese,A., Boija,A., Klein,I.A., Coffey,E.L., Shrinivas,K., Abraham,B.J., Hannett,N.M., Zamudio,A.V., Manteiga,J.C., Li,C.H., Guo,Y.E., Day,D.S., Schuijers,J., Vasile,E., Malik,S., Hnisz,D., Lee,T.I., Cisse,I.I., Roeder,R.G., Sharp,P.A., Chakraborty,A.K., and Young,R.A. (2018) Coactivator condensation at super-enhancers links phase separation and gene control. Science, 361, eaar3958.

7. Wei,M.T., Chang,Y.C., Shimobayashi,S.F., Shin,Y., Strom,A.R., and Brangwynne,C.P. (2020) Nucleated transcriptional condensates amplify gene expression. Nat. Cell Biol., 22, 1187-1196.

8. Hilbert,L., Sato,Y., Kuznetsova,K., Bianucci,T., Kimura,H., Julicher,F., Honigmann,A., Zaburdaev,V., and Vastenhouw,N.L. (2021) Transcription organizes euchromatin via microphase separation. Nat. Commun., 12 1360

9. Nakagawa,S., Yamazaki,T. and Hirose,T. (2018) Molecular dissection of nuclear paraspeckles: towards understanding the emerging world of the RNP milieu. Open Biol., 8, 180150.

10. Chujo,T., Yamazaki,T. and Hirose,T. (2015) Architectural RNA (arcRNAs): A class of long noncoding RNAs that function as the scaffold of nuclear bodies. Biochim. Biophys. Acta, 1859, 139-146.

11. Yamazaki,T., Nakagawa,S. and Hirose,T. (2019) Architectural RNAs for Membraneless Nuclear Body Formation. Cold Spring Harb. Symp. Quant. Biol., 84, 227-237.

12. Banani,S.F., Lee,H.O., Hyman,A.A. and Rosen,M.K. (2017) Biomolecular condensates: organizers of cellular biochemistry. Nat. Rev. Mol. Cell Biol., 18, 285-298.

13. Shin,Y. and Brangwynne,C.P. (2017) Liquid phase condensation in cell physiology and disease. Science, 357, eaaf4382.

14. Doi,M. (2013) Soft Matter Physics. Oxford University Press, Oxford, UK.

15. Feric,M., Vaidya,N., Harmon,T.S., Mitrea,D.M., Zhu,L., Richardson,T.M., Kriwacki,R.W., Pappu,R.V., and Brangwynne,C.P. (2016) Coexisting Liquid Phases Underlie Nucleolar Subcompartments. Cell, 165, 1686-1697.

16. Ide,S., Imai,R., Ochi,H. and Maeshima,K. (2020) Transcriptional suppression of ribosomal DNA with phase separation. Sci. Adv., 6, eabb5953.

17. Maiser,A., Dillinger,S., Langst,G., Schermelleh,L., Leonhardt,H. and Nemeth,A. (2020) Article title. Sci. Rep., 10, 7462.

18. Yao,R.W., Xu,G., Wang,Y., Shan,L., Luan,P.F., Wang,Y., Wu,M., Yang,L.Z., Xing,Y.H., Yang,L. and Chen,L.L. (2019) Nascent Pre-rRNA Sorting via Phase Separation Drives the Assembly of Dense Fibrillar Components in the Human Nucleolus. Mol. Cell, 76, 767-783.

19. Stasevich,T.J., Hayashi-Takanaka,Y., Sato,Y., Maehara,K., Ohkawa,Y., Sakata-Sogawa, K., Tokunaga,M., Nagase,T., Nozaki,N., McNally,J.G. and Kimura,H. (2014) Regulation of RNA polymerase II activation by histone acetylation in single living cells. Nature, 516, 272-275.

20. Alexander,S. (1980) Adsorption of chain molecules with a polar head a scaling description. J. Phys. France, 38, 983-987.

21. de Gennes,P.G. (1980) Conformation of Polymers Attached to an Interface. Macromolecules, 13, 1069-1075.

22. Daoud,M. and Cotton,J.P. (1982) Star shaped polymers: a model for the conformation and its concentration dependence. J. Phys. France, 43, 531538 .

23. Safran,S.A. (2003) Statistical Thermodynamics of Surfaces, Interfaces, and Membranes. Westview Press, CO, US

24. Doi,M. (1996) Introduction to Polymer Physics. Oxford Univ. Press, NY,
US.

25. de Gennes,P.G.(1979) Scaling Concepts in Polymer Physics. Cornell Univ. Press, NY, US.

26. Peltonen,K., Colis,L., Liu,H., Trivedi,R., Moubarek,M.S., Moore,H.M., Bai,B., Rudek,M.A., Bieberich,C.J., and Laiho,M. (2014) A Targeting Modality for Destruction of RNA Polymerase I that Possesses Anticancer Activity. Cancer Cell, 25, 77-90.

27. Nicolas,E., Parisot,P., Pinto-Monteiro,C., de Walque,R., De Vleeschouwer, C. and Lafontaine,D.L.J. (2016) Involvement of human ribosomal proteins in nucleolar structure and p53-dependent nucleolar stress.. Nat. Comm., 7, 11390.

28. Cochard,A., Navarro,M.G.J., Kashida,S., Kress,M., Weil,D. Gueroui,Z. (2021) RNA at the surface of phase separated condensates impacts their size and number. bioRxiv, doi https://doi.org/10.1101/2021.06.22.449254

29. Ou-Yang,Z.C., Ji-Xing,L., and Zhang,X.Y. (1999) Geometric Methods in the Elastic Theory of Membranes in Liquid Crystal Phases. World Scientific Publishing, Co. Ltd, Singapore.

30. Yamamoto,T. and Schiessel,H. (2019) Dilution of contact frequency between superenhancers by loop extrusion at interfaces. Soft Matter, 15, 7635-7643.

31. Hori,Y., Shimamoto,A. and Kobayashi,T. (2021) The human ribosomal RNA gene is composed of highly homogenized tandem clusters. bioRxiv, doi: https://doi.org/10.1101/2021.06.02.446762.

32. Yamamoto,T., Yamazaki,T. and Hirose,T. (2020) Phase separation driven by production of architectural RNA transcripts. Soft Matter, 16, 4692 4698.

33. Wu,M., Xu,G., Han,C., Luan,P.F., Xing,Y.H., Nan,F., Yang,L.Z., Huang,Y., Yang,Z.H., Shan,L., Yang,L., Liu,J. and Chen,L.L. (2021) IncRNA SLERT controls phase separation of FC/DFCs to facilitate Pol I transcription. Science, 2, 283.

34. Henninger,J.E., Oksuz,O., Shrinivas,K., Sagi,I., LeRoy,G., Zheng,M.M., Andrews,J.O., Zamudio,A.V., Lazaris,C., Hannett,N.M., Lee T.I., Sharp,P.A., Cisse,I.I., Chakraborty,A.K., and Young,R.A. (2021) RNAMediated Feedback Control of Transcriptional Condensates. Cell, 184 207-225.

35. Lamond,A.I. and Spector,D.L. (2003) Nuclear speckles: a model for nuclear organelles. Nat. Rev. Mol. Cell Biol., 4, 605-612.

36. Yamazaki,T., Yamamoto, T., Yoshino,H., Souquere,S., Nakagawa,S., Pierron,G., and Hirose,T. (2021) Paraspeckles are constructed as block copolymer micelles EMBO J, 40, e107270.

37. Yamamoto,T., Yamazaki,T., and Hirose,T. (2020) Triblock copolymer micelle model of spherical paraspeckles. bioRxiv, doi: https://doi.org/10.1101/2020.11.01.364190.

38. Yamazaki,T., Souquere,S., Chujo,T., Kobelke,S., Chong,Y.S., Fox,A.H Bond,C.S., Nakagawa,S., Pierron,G., and Hirose,T. (2018) Functional Domains of NEAT1 Architectural IncRNA Induce Paraspeckle Assembly through Phase Separation. Molecular Cell, 70, 1038-1053.

39. Hirose,T., Virnicchi,G., Tanigawa,A., Naganuma,T., Li,R., Kimura,H., Yokoi,T., Nakagawa,S., Bénard,M., Fox,A.H., and Pierron,G., (2014) NEAT1 long noncoding RNA regulates transcription via protein sequestration within subnuclear bodies. Mol. Biol. Cell, 25, 169-183.

40. Kawaguchi,T., Tanigawa,A., Naganuma,T., Ohkawa,Y., Souquere,S., Pierron,G., and Hirose,T. (2015) SWI/SNF chromatin-remodeling complexes function in noncoding RNA-dependent assembly of nuclear bodies.. Proc. Natl. Acad. Sci. USA, 112, 4304-4309.

41. Aly,M.K., Ninomiya,K., Adachi,S., Natsume,T., and Hirose,T. (2019) Two distinct nuclear stress bodies containing different sets of RNAbinding proteins are formed with HSATIII architectural noncoding RNAs upon thermal stress exposure.. Biochem. Biophys. Res. Co., 516, 419-423.

42. Biamonti,G. and Vourc'h,C. (2010) Nuclear Stress Bodies. Cold Spring Harb. Perspect. Biol., 2, a000695.

43. Ninomiya,K. and Hirose,T. (2020) Short Tandem Repeat-Enriched Architectural RNAs in Nuclear Bodies: Functions and Associated Disease. non-coding RNA, 6, 6. 